\title{
Disequality Management in Integer Difference Logic via Finite Instantiations*
}

\author{
Hyondeuk Kim \\ Hoonsang Jin \\ Fabio Somenzi \\ Department of Electrical and Computer Engineering, \\ University of Colorado at Boulder, CO 80309-0425
}

hyondeuk.kim@colorado.edu

hoonsang.jin@samsung.com

fabio@colorado.edu

\begin{abstract}
The last few years have seen the advent of a new breed of decision procedures for various fragments of first-order logic based on propositional abstraction. A lazy satisfiability checker for a given fragment of first-order logic invokes a theory-specific decision procedure (a theory solver) on a (partial) model for the abstraction. If the model is found to be consistent in the given theory, then a model for the original formula has been found. Otherwise, a refinement of the propositional abstraction is extracted from the proof of inconsistency and the search is resumed. We describe a theory solver for integer difference logic that is effective when the formula to be decided contains equality and disequality (negated equality) constraints so that the decision problem partakes of the nature of the pigeonhole problem. We propose a reduction of the problem to propositional satisfiability by computing bounds on a sufficient subset of solutions, and present experimental evidence for the efficiency of this approach.
\end{abstract}

KEYWORDS: theorem prover, SAT solver, difference logic, finite instantiations

Submitted November 2006; revised May 2007; published July 2007

\section{Introduction}

Decision procedures for fragments of first-order logic have been the subject of intense scrutiny in the last few years. On the one hand, emerging applications like model checking of infinite state systems rely on such decision procedures for tasks like predicate abstraction [1]. On the other hand, algorithmic advances have significantly increased the range of problems that can be tackled, and hence have stimulated interest.

In particular, dramatic increase in performance of propositional satisfiability (SAT) solvers has led to the development of decision procedures that rely on the propositional abstraction of formulae from more expressive logics like the logic of linear arithmetic constraints, Presburger arithmetic, or the logic of equality and uninterpreted function symbols (EUF). The propositional abstraction of a formula is obtained by replacing the atomic formulae of the specific theory (e.g., $x-y \leq 5$ or $f(x)=f(y)$, where $f$ is an uninterpreted function symbol) with fresh propositional variables. Each model of the abstraction maps to a conjunction of literals in the original formula that can be checked for consis-

* Supported by SRC contract 2006-TJ-1365. 
tency with theory-specific procedures. If such a procedure establishes consistency, then the given formula is satisfiable and the enumeration terminates. Otherwise, from the proof of inconsistency a refinement of the propositional abstraction is extracted and the search is resumed.

There are several ways to combine the propositional reasoning engine with the theoryspecific procedures. One broad classification is into lazy and eager approaches. A lazy solver produces an initial propositional approximation that is concise and possibly quite coarse; it relies on refinements during the enumeration of solutions. By contrast, an eager solver adds constraints to the initial propositional abstraction that embody known relationships among the literals. An example is given by the constraints that encode transitivity of equality. The most effective solvers often adopt elements of both approaches and tailor their strategies to the theory (theories) at hand.

In this paper we focus on Integer Difference Logic (IDL), in which arithmetic atomic formulae constrain the difference between the values of pairs of integer variables. This logic finds extensive application to problems involving timing and scheduling constraints, resource allocation, and program analysis. IDL is closely related to Real Difference Logic (RDL), to the point that a decision procedure for the latter based on propositional abstraction also works for the former, as long as the coefficients are integers. It is sufficient to rewrite each equality constraint (of the form $x-y=n$ ) as the conjunction of two inequalities. However, if an equality constraint is negated, then the conjunction turns into a disjunction, which requires case splitting in the enumeration of the propositional solutions. In contrast, we propose an approach that does not decompose equalities and their negations; rather, it converts the problem of checking satisfiability of a conjunction of arithmetic atomic formulae into a set of propositional satisfiability checks - whose cardinality is bounded by the number of strongly connected components (SCC) of a suitable constraint graph.

The conversion to propositional satisfiability that we propose is based on the ability to bound the values of the integer variables that appear in the formula. While in general such variables are not bounded, we show that to decide satisfiability of a set of constraints whose graph is a single SCC it is sufficient to consider a subset of the solutions for which bounds are easily established. We also show how the general case can be efficiently solved given solutions for the individual SCCs of the constraint graph. Experiments show that our new approach, which combines techniques typical of both the lazy and the eager approaches, greatly improves the efficiency of our decision procedure for problem instances in which disequalities play a significant role, and makes it very competitive with respect to state-ofthe-art tools.

This paper is an extended verision of [17] and is organized as follows: Section 2 reviews background and introduces notation. Section 3 and Section 4 discuss the minimizing the abstract models and the bounds on solutions, while Sect. 5 deals with the implementation of our theory solver. After a survey of related work in Sect. 6, experiments are presented in Sect. 7, and conclusions are offered in Sect. 8.

\section{Preliminaries}

Let $P$ be a set of propositional variables and $X$ a set of integer-valued variables. We define inductively integer difference logic (IDL) formulae as follows. 
- $p \in P$ is a (propositional atomic) IDL formula.

- $x-y \leq n$ and $x-y=n$ are (arithmetic atomic) IDL formulae, for $x, y \in X, n \in Z$.

- If $\varphi$ and $\psi$ are IDL formulae, so are $\varphi \wedge \psi$ and $\neg \varphi$.

The following abbreviations are also defined:

$$
\begin{array}{rlrl}
x-y<n & \doteq x-y \leq n-1 & x-y \neq n & \doteq \neg(x-y=n) \\
x=y & \doteq(x-y=0) & x \neq y & \doteq \neg(x=y) .
\end{array}
$$

A literal is an atomic formula, or the negation of an atomic formula. A clause is the disjunction of a set of literals, and a formula in conjunctive normal form (CNF) is the conjunction of a set of clauses. Note that $x-y=n$ could be defined as an abbreviation $((x-y \leq n) \wedge(x-y \geq n))$. We choose not to do so to stress that our algorithm does not split equalities or disequalities (as mentioned in Sect. 1) and also to keep the definition of clausal formulae simple.

A (partial) interpretation for an IDL formula $\varphi$ is a pair of (partial) functions $\alpha: X \rightarrow Z$ and $\beta: P \rightarrow\{$ false,true $\}$. If (partial) interpretation $(\alpha, \beta)$ is such that replacing each variable $x \in X$ with $\alpha(x)$, and every variable $p \in P$ with $\beta(p)$ in $\varphi$ produces a true statement, it is called a (partial) model. A formula is satisfiable if it has a model, and is valid if every interpretation is a model. If a conjunction of literals $\varphi$ is not satisfiable, then a (minimal) explanation for the unsatisfiability of $\varphi$ is the conjunction of a (minimal) subset of the literals in $\psi$ that is not satisfiable.

Propositional logic is the fragment of IDL obtained by omitting the rule that defines arithmetic atomic formulae. Efficient algorithms to decide the satisfiability of propositional logic formulae are based on the DPLL procedure $[7,6]$, and exploit techniques like clause recording, conflict analysis, nonchronological backtracking, and fast Boolean constraint propagation [26, 22].

In recent times, decision procedures for IDL, and other fragments of quantifier-free firstorder logic, have been based on the DPLL procedure as well. Given a set of propositional variables $B$ such that $B \cap P=\emptyset$, one obtains a propositional formula $\varphi^{b}$ from an IDL formula $\varphi$ by replacing each arithmetic atomic subformula of $\varphi$ with a distinct variable from $B$. The resulting formula $\varphi^{b}$ is unsatisfiable only if $\varphi$ is unsatisfiable. Each model of $\varphi^{b}$ corresponds to a conjunction of literals of $\varphi$. Given a decision procedure for the conjunction of arithmetic atomic propositions in IDL (a theory solver), one therefore derives a complete decision procedure for IDL by enumerating the models of $\varphi^{b}$, extracting from each of them the corresponding conjunction of arithmetic atomic propositions and their negations, and checking these conjunctions for satisfiability using the theory solver. In the following, we refer to the conjunction of a set of arithmetic literals as a set of IDL constraints.

The theory solver rewrites the IDL constraints to be checked according to their form:

1. $x-y \leq n$ : unchanged;

2. $x=y$ : unchanged;

3. $x-y=n$, with $n \neq 0$ : split into $(x-y \leq n) \wedge(y-x \leq-n)$; 
4. $\neg(x-y \leq n)$ : rewritten as $y-x \leq-n-1$;

5. $\neg(x=y)$ : rewritten as $x \neq y$;

6. $\neg(x-y=n)$, with $n \neq 0$ : rewritten as $x-y \neq n$.

Constraints of type 1, 3, and 4 are inequalities $(I)$. Constraints of type 2 are equalities $(Q)$, and finally, constraints of type 5 and 6 are disequalities $(D)$. Specifically, constraints of type 5 form the set $D_{0} \subseteq D$. Let $C=I \cup Q \cup D$.

An edge integer-labeled directed graph is a triple $G=(V, E, \lambda)$, where $V$ is a set of vertices, $E \subseteq V \times V$ is a set of edges, and $\lambda: E \rightarrow Z$ is an edge labeling function. A strongly connected component (SCC) of $G$ is a maximal subgraph $G^{\prime}$ of $G$ such that every two nodes of $G^{\prime}$ are connected by a path in $G^{\prime}$. An SCC is trivial if it consists of one vertex and no arcs. The SCCs of $G$ define a partition of $V$. The $S C C$ quotient graph $\widehat{G}=(\widehat{V}, \widehat{E})$ of $G$ is a directed acyclic graph with one vertex for each SCC of $G$ and an edge $(A, B) \in \widehat{E}$ if and only if there exist $x \in A$ and $y \in B$ such that $(x, y) \in E$.

Given a distinguished source vertex $s \in V$, distances of all vertices from $s$ are well defined provided there exists no negative cycle in $G$; that is, no cycle such that the sum of the labels on the edges along the cycle is negative. The Bellman-Ford algorithm [5] reports negative cycles if they are present, and computes the distance $\delta(x)$ of each vertex in $V$ from the source $s$ otherwise. The slack of an edge $(x, y) \in E$ is given by $\sigma((x, y))=\lambda((x, y))-(\delta(y)-\delta(x))$. It is easy to see that for all $e \in E, \sigma(e) \geq 0$ and that $\sigma((x, y))=0$ if and only if $(x, y)$ is on a shortest path from $s$ to $y$ in $G$. Distances and slacks obviously depend on the choice of source vertex.

Given a (finite) set $I$ of inequality constraints (i.e., of the form $x-y \leq n$ ), their constraint graph $G=(V, E, \lambda)$ is a labeled directed graph defined as follows:

- $V \subseteq X$ is the set of variables appearing in the constraints in $I$.

- There is an $\operatorname{arc}(x, y) \in E$ with $\lambda((x, y))=n$ if and only if there is a constraint $y-x \leq n$ in $I$.

It is well known [5] that $I$ is satisfiable if and only if $G$ contains no negative cycle. In fact, adding both sides of the constraints forming a cycle of length $w$, one gets $0 \leq w$, which is not satisfiable when $w<0$. If, on the other hand, no negative cycle exists in $G$, then one can find a model for $I$ by solving a single-source shortest-path problem on an augmented graph $G_{a}$, obtained from $G$ by adding a new reference vertex $x_{r}$ and $\operatorname{arcs}$ labeled 0 from $x_{r}$ to all the other vertices. Let $\delta(x)$ be the distance of $x \in V$ from $x_{r}$ in $G_{a}$. Then $\delta$ is a model for $I$. It is also well known that, given a model of $I, \alpha: V \rightarrow Z$, and a constant, $c \in Z$, the interpretation $\alpha^{\prime}: V \rightarrow Z$ defined by $\alpha^{\prime}(x)=\alpha(x)+c$ is also a model of $I$, because $\alpha^{\prime}(x)-\alpha^{\prime}(y)=\alpha(x)-\alpha(y)$. This observation allows an easy encoding of range constraints in IDL. A set of constraints $\left\{l_{i} \leq x_{i} \leq u_{i}\right\}$ is translated to $\left\{x_{i}-y \leq u_{i}\right\} \cup\left\{y-x_{i} \leq-l_{i}\right\}$, where $y$ is a fresh variable. The solution $\alpha$ obtained from the constraint graph is then translated so that $\alpha^{\prime}(y)=0$. One fresh variable suffices for multiple range constraints.

Since integer labels imply integer distances, if the right-hand sides of the constraints are integer-valued, and the constraints are satisfiable when the variables range over the real numbers, then an integer-valued solution is also guaranteed to exist. Loosely speaking, the 
satisfiability problem for inequalities is the same for IDL and real difference logic (RDL). Adding equality constraints to the inequalities does not change this state of affairs: Given a constraint $x-y=n$, one replaces $x$ by $y+n$; if no immediate inconsistencies arise, one continues with the construction of the constraint graph. In contrast, if disequality constraints (i.e., negations of equalities) are allowed, an unsatisfiable conjunction of IDL constraints may be satisfiable when regarded as an RDL formula. An example is given by $\bigwedge_{1 \leq i \leq p}\left(1 \leq x_{i} \leq h\right) \wedge \bigwedge_{1 \leq i<j \leq p}\left(x_{i} \neq x_{j}\right)$, which exemplifies the pigeonhole principle. ${ }^{1 .}$

\section{Minimizing the Abstract Models}

Given the set of clauses $\varphi^{b}$ and a complete model for them produced by the propositional reasoning engine, we consider now the problem of identifying a minimal (partial) model such that at least one literal for each clause is true. The intent of finding such minimal model is twofold: to alleviate the task of the theory solver and to make the exploration of the models of $\varphi^{b}$ more efficient. A greedy solution to our problem is easily obtained by considering each variable in turn and removing it from the model if no clause becomes unsatisfied as a result. We now describe how such a solution can be implemented efficiently in the context of the algorithm that enumerates the solutions to the propositional abstraction. That is, we show how we can take advantage of the information gathered by the propositional SAT solver to significantly speed up the choice of the minimal model.

Two observations from [25] provide the foundation for our method. The first is that no variable that received its value by implication (rather than decision) by the SAT solver can be removed from the model. This fact greatly reduces the number of variables that are candidates for removal. The second observation concerns the list of watched literals and assumes that only two literals are watched by the SAT solver [22]. It can then be shown that when a complete model is found, at least one watched literal in every clause is true. Therefore, when considering a variable for removal it is sufficient to check if it provides the only true literal in the clauses in which the satisfied literal of the variable is watched. The clauses in which that literal is not watched can be safely ignored. Moreover, conflict clauses recorded by the SAT solver do not need to be examined because they are known to be satisfied whenever the original clauses are satisfied.

When a clause in which the candidate literal is watched is examined, a substitute literal that is true is sought so as to maintain the invariant. If there is no substitute and the other watched literal is false, the candidate is rejected. On the other hand, if this process manages to empty the watched-literal list of the candidate (except possibly for conflict clauses), the candidate is removed from the model.

The effect of the minimization procedure is to alter the watched-literal lists of the solver. However, the enumeration process can resume from the modified lists without any adverse consequence. The algorithm that we have described runs in polynomial time, but only guarantees a minimal set of variables. Reduction from set covering shows that deciding whether a model of size $k$ exists for a set of propositional clauses is NP-complete.

The order in which literals are considered for removal depends on the constraints they represent. The check for consistency of a set of constraints tends to be easier if disequal-

1. This does not contradict what was observed in Sect. 1 because $x \neq y$ translates into $(x<y) \vee(y<x)$ for RDL, but translates into $(x \leq y-1) \vee(y \leq x-1)$ for IDL. 
ity constraints are the first candidates for elimination. They are followed by inequality constraints, and finally equality constraints, in that order.

\section{Bounds on Solutions}

In this section we show how bounds to the solutions of a set of constraints are computed and how those bounds are used in checking for consistency of (partial) interpretations of $\varphi^{b}$. Two cases must be distinguished depending on whether the interpretation to be checked is known to be a model of $\varphi^{b}$ : If it is not known to be a model, a cheap check is applied, which can only report inconsistency. Otherwise, a more expensive, complete check is applied in addition, which decides consistency and computes a model of $\varphi$ if it exists.

\subsection{Bound Computation}

It was recalled in Sect. 2 that from a solution $\alpha$ to a set of inequality constraints, one can derive a family of solutions $\{\alpha+c\}$. In general, however, not all solutions are obtained one from the other by translation. Consider the constraints $\{(x-y \leq 1),(y-x \leq 0)\}$. The two interpretations $\alpha_{1}(x)=0, \alpha_{1}(y)=0$ and $\alpha_{2}(x)=1, \alpha_{2}(y)=0$ satisfy the constraints, though there is no $c$ such that $\alpha_{1}=\alpha_{2}+c$. Such solutions are called independent. In general, there may be several families of independent solutions, and therefore, multiple distinct solutions that assign a given value to a distinguished variable. The following result characterizes these sets of solutions and forms the basis for our treatment of disequality constraints in IDL.

Theorem 1. Let I be a set of inequality constraints. Let $G=(V, E, \lambda)$ be the constraint graph associated to $I$. Suppose that $G$ contains no negative cycle and consists of one $S C C$. Let $\delta_{a b}$ be the distance from a to $b$ in $G$. For $x \in V$ and $n \in Z$, let $S_{x}^{n}$ be the set of solutions $\alpha: V \rightarrow Z$ to $I$ such that $\alpha(x)=n$. Then, for each vertex $y \in V$, there exist bounds $y_{l}=n-\delta_{y x}$ and $y_{u}=n+\delta_{x y}$, such that for every solution in $S_{x}^{n}, y_{l} \leq \alpha(y) \leq y_{u}$.

Proof. By definition of SCC, every vertex in $V$ is reachable from $x$ in $G$; likewise, $x$ is reachable from any vertex in $G$. Let $\delta_{x y}$ be the distance of $y$ from $x$ (the length of a shortest path). Such a distance is defined because there are no negative cycles in $G$. Adding both sides of all the constraints along the path yields $y-x \leq \delta_{x y}$. Therefore, for every solution $\alpha \in S_{x}^{n}$, it must be $\alpha(y) \leq n+\delta_{x y}$. Said otherwise, $y_{u}=n+\delta_{x y}$. For the lower bound, if $\delta_{y x}$ be the distance of $x$ from $y$ in $G$, then, for every solution $\alpha \in S_{x}^{n}$, it must be $\alpha(y) \geq n-\delta_{y x}$; that is, $y_{l}=n-\delta_{y x}$.

Satisfaction of disequalities is not affected by translation. Therefore, a set of constraints including both inequalities and disequalities is satisfiable if and only if there exists a solution $\alpha$ such that $\alpha(x)=n$. This allows us to limit the search to the set $S_{x}^{n}$ for an arbitrarily chosen $n$. Theorem 1 asserts that solutions in this set are bounded. The way this result is exploited depends on whether the set of constraints corresponds to a model of $\varphi^{b}$. The next two subsections discuss the two cases. 


\subsection{Inconsistency Check for Partial Interpretations}

Given a partial abstract interpretation that is not known to be a model of $\varphi^{b}$, we want to check the corresponding constraints for inconsistency to prune the search space (as in theory propagation) or to possibly avoid the more expensive check of Section 4.3. A set of constraints is assumed to be given along with ranges for every variable in them. It is also assumed that the graph has one SCC. If that is not the case, each SCC is checked in turn: The constraints are inconsistent if at least one SCC is inconsistent. Though the check described in the next section could be applied in this case, we are interested in a cheaper criterion.

The quick check for inconsistency is based on two observations: The first is that if all variables in the SCC have the same range, then the disequalities define a graph whose chromatic number must not exceed the size of the range for the constraints to be satisfiable. (The chromatic number is the least number of colors needed to assign different colors to adjacent vertices in the graph.) The second observation is that the chromatic number of a graph is bounded from below by the size of a clique of the graph and from above by the number of vertices. From these observations, it is easy to prove the following theorem.

Theorem 2. Let $D_{0}$ be a set of disequality constraints of the form $x_{i}-x_{j} \neq 0$. Let $X=\left\{x_{1}, \ldots, x_{n}\right\}$ be the set of variables in $D_{0}$. Let $L=\left\{l_{1}, \ldots, l_{n}\right\} \in Z^{n}$ and $U=$ $\left\{u_{1}, \ldots, u_{n}\right\} \in Z^{n}$ be the bounds on the variables in $X\left(l_{i} \leq x_{i} \leq u_{i}\right)$. For $y_{l}, y_{u} \in Z$, let $\Gamma=\left\{\gamma_{1}, \ldots, \gamma_{p}\right\}$ be the subset of $X$ such that

$$
\Gamma=\left\{x_{i} \in X \mid y_{l} \leq l_{i} \wedge u_{i} \leq y_{u}\right\}
$$

Let $\rho=y_{u}-y_{l}+1$. Let $G_{D}=(V, E)$ be the disequality graph associated to $D_{0}$, such that $V=\left\{v_{1}, \ldots, v_{n}\right\}$ and $\left\{v_{i}, v_{j}\right\} \in E$ if and only if $x_{i}-x_{j} \neq 0 \in D_{0}$ or $x_{j}-x_{i} \neq 0 \in D_{0}$. If $G_{D}$ contains a clique of size greater than $\rho$ then $D_{0}$ is inconsistent.

Example 1. Consider the set of disequality constraints $D_{0}=\{(x-y \neq 0),(y-z \neq$ $0),(z-x \neq 0)\}$ with variables $y, z$ that have the same range, $0 \leq y, z \leq 1$, and variable $x$ that has range $0 \leq x \leq 0$ which is a subset of the common range. Let $y_{l}=0$ and $y_{u}=1$; then $\Gamma=\{x, y, z\}$. A clique consisting of variables $x, y, z$ is present in $G_{D}$. Since $|\Gamma|=3>2=\rho$, the constraints are inconsistent. An explanation of inconsistency consists of the disequality constraints $\{(x-y \neq 0),(y-z \neq 0),(z-x \neq 0)\}$ and the inequality constraints that generated the range, $0 \leq y, z \leq 1,0 \leq x \leq 0$.

The check based on Theorem 2 results in one of three outcomes: A suitable clique has been found and inconsistency is declared; a large enough clique was not found because of the heuristic nature of the algorithm; a large enough clique is known not to exist. In the first case, an explanation of inconsistency is derived from the disequalities forming the clique and the inequalities responsible for the bounds. In the last two cases, the result is inconclusive, because the chromatic number of a graph can be arbitrarily larger than the size of the largest cliques. However, if a large enough clique does not exist in the graph, and the interpretation is not known to be a model, we avoid a full check for inconsistency, which is rather expensive and likely to fail. (If the interpretation is a model, on the other hand, the consistency check must be performed for the whole decision procedure to be sound.) 


\subsection{Consistency Check for Abstract Models}

If the constraints correspond to a model of $\varphi^{b}$, we want to decide consistency and compute a model of $\varphi$ in case the answer is affirmative. For this, we resort to finite instantiation. Specifically, we can encode each integer variable with enough binary variables to span its range and translate the satisfiability problem for a conjunction of inequality and disequality constraints into a propositional satisfiability problem.

Theorem 1 applies when the constraint graph consists of one SCC. If that is not the case, we examine the SCC quotient graph one SCC at the time. If there is no negative cycle in the constraint graph $G$, the only reason for unsatisfiability is the inability to satisfy the disequalities within some SCC of $G$. Therefore, if the finite instantiation of each SCC is satisfiable, the entire set of constraints is satisfiable. This can be shown as follows.

Let $G$ be the constraint graph. Extend $G$ by adding one edge for every disequality constraint $x-y \neq n$ (where $n$ may be 0 ) such that $x$ and $y$ belong to different SCCs. Let $\preceq$ be the preorder defined by $u \preceq v$ if there is a path in $G$ from $u$ to $v$. (The preorder is updated after each edge addition.) If $x \preceq y$, add $y-x \leq-n-1$ to $E$; if $y \preceq x$, add $x-y \leq n-1$. If $x$ and $y$ are not comparable in the preorder, add either $y-x \leq-n-1$ or $x-y \leq n-1$, but not both. Note that adding these edges does not create cycles, and therefore does not change the SCCs of $G$. (See Sect. 5.)

Let $\widehat{G}=(\widehat{V}, \widehat{E})$ be the SCC quotient graph of the extended $G$. Consider the vertices in $\widehat{V}$ starting from the minimal SCCs (those with no predecessors) and proceeding in a chosen topological order. Let $A_{i}$ be the $i$-th SCC in that order and let $\alpha_{i}$ be a solution for the constraints corresponding to its edges. Inductively assume that $\beta_{i-1}$ is a solution for the constraints in the subgraph induced by $\bigcup_{0<j<i} A_{j}$. Let $k$ be the maximum amount by which any constraint corresponding to an edge into $A_{i}$ is violated. (Let $k=0$ if no such violation exists.) Finally, let $\alpha_{i}^{\prime}=\alpha_{i}-k$. Then, $\beta_{i}=\beta_{i-1} \cup \alpha^{\prime}$ is a solution for the constraints in the subgraph induced by $\bigcup_{0<j \leq i} A_{i}$.

\section{Algorithm}

We assume a decision procedure for IDL based on propositional abstraction. The given IDL formula $\varphi$ is translated into a propositional formula $\varphi^{b}$ as described in Sect. 2. A propositional reasoning engine enumerates the models to $\varphi^{b}$ and calls the theory solver to determine whether that abstract model corresponds to a consistent interpretation of the integer-valued variables.

The theory solver for IDL is relatively efficient. Therefore, it is advantageous to call it also on a partial interpretations to terminate the fruitless search of part of the state space, or to learn so-called theory consequences [23]. Our implementation follows this approach, though the equality constraints $(x-y=n \neq 0)$ are split and the full check for inconsistencies due to disequalities is applied only to abstract models. (See lines 38-42 of Fig. 1.) We omit the details of the incremental implementation of the Bellman-Ford algorithm. The interested reader is referred to [30]. 


\subsection{The Theory Solver}

The theory solver is called with a collection of arithmetic literals whose corresponding propositional literals are true in a (partial) interpretation of the propositional formula $\varphi^{b}$; it then decides whether there is an interpretation to the integer-valued variables that satisfies the conjunction of all those literals. The first step is to obtain a set of arithmetic atomic formulae (without negations) from the given set of literals. The given literals are rewritten and divided into $Q, I$, and $D$ as described in Sect. 2 .

The theory solver, whose pseudocode is shown in Figures 1 and 2, adopts the layered approach of MathSAT [4]. For IDL, it considers three main layers: equalities, inequalities, and disequalities. Let $X=\subseteq X$ be the set of integer-valued variables appearing in the equalities in $Q$. The theory solver creates an undirected equality graph $Q=\left(X_{=}, \Gamma\right)$, where

$$
\Gamma=\left\{\left\{x_{i}, x_{j}\right\}: x_{i}=x_{j} \in Q\right\}
$$

The vertices of $Q$ are in the same class if they are made equivalent by the equality constraints. The feasibility of $Q$ with $D_{0}$ is checked by comparing the equivalence class of the two vertices of each disequality constraint in $D_{0}$. If two vertices are in the same class, an explanation of infeasibility is returned. If the set of equality constraints is feasible, the variables in the same class are merged into a single variable, and some simplified constraints in $D$ and $I$ are dropped from the set.

The algorithm continues by checking the feasibility of the set of inequality constraints. Let $V \subseteq X$ be the set of integer-valued variables appearing in $I$. The theory solver creates a constraint graph $G=(V, E, \lambda)$ from $I$ as explained in Sect. 2. The Bellman-Ford algorithm is run on $G$. If a negative cycle is found, the set $I$ is infeasible; a negative cycle with a subset of $Q$ provides the explanation of infeasibility. Equality constraints are involved in the explanation if the constraints on the negative cycle were obtained by simplification in the equality layer. If there is no negative cycle in $G$, the set $I \cup Q$ is feasible; therefore a solution $\delta: V \rightarrow Z$ is returned by the Bellman-Ford algorithm. ${ }^{2}$.

The (simplified) set $I$ combined with $D$ is considered in the next step. Let $G_{0}$ be the subgraph of $G$ such that the edges with non-zero slacks for solution $\delta$ are removed from $G$. Since the slacks of the edges of $G_{0}$ are zero, the difference between the values of two variables in the same SCC of $G_{0}$ is the same in all solutions to the constraints. In fact, each cycle in $G_{0}$ is of length 0 [19]; hence, if $x$ and $y$ are on one cycle of $G_{0}$ and the distance from $x$ to $y$ along the cycle is $k$, then the distance from $y$ to $x$ is $-k$. It follows that every solution to $I$ must satisfy $y-x \leq k$ and $x-y \leq-k$, that is, $y-x=k$. In other words, an SCC of $G$ such that its vertex set induces also an SCC of $G_{0}$ has only one family of solutions. (See Sect. 4.)

Each disequality constraint $d \in D$ is checked for feasibility against each SCC of $G_{0}$. If the two variables $x, y$ in $x-y \neq n$ (where $n$ may be 0 ) are in the same SCC of $G_{0}$ and $\delta(x)-\delta(y)=n$, then the set $I \cup Q \cup D$ is infeasible. The violated disequality $d$, together with a cycle that contains $x$ and $y$ and an appropriate subset of $Q$ constitutes the explanation of infeasibility. If the two variables $x$ and $y$ in $d$ are in the same SCC of $G_{0}$ and $\delta(x)-\delta(y) \neq n$, then $d$ is redundant and is dropped from $D$. Disequalities connecting variables in different

2. The algorithm is, in principle, applied to the augmented graph $G_{a}$ described in Sect. 2. In practice, no augmentation of $G$ is required: it suffices to initialize all distances to 0 . 


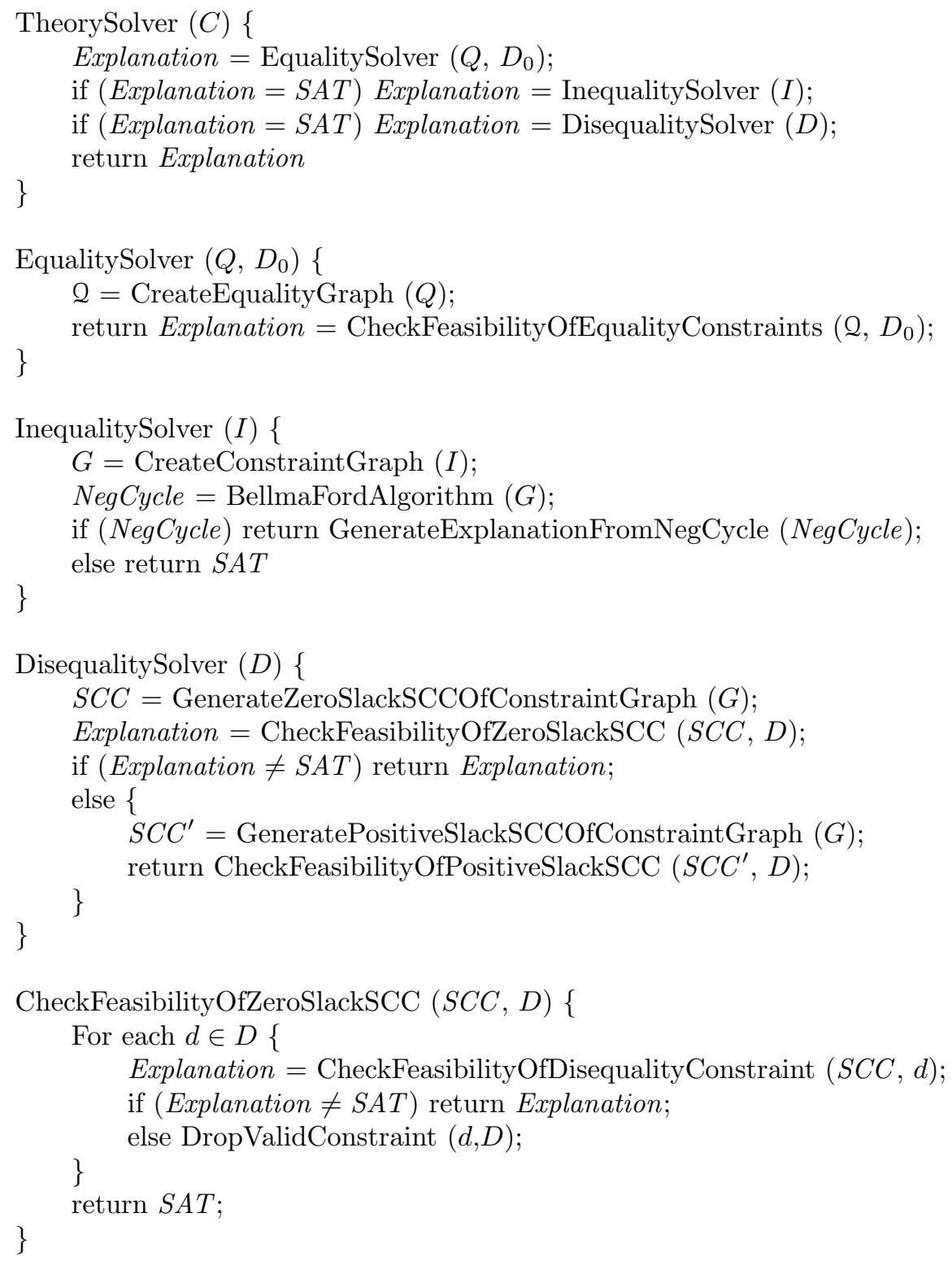

Figure 1. Theory solver algorithm 


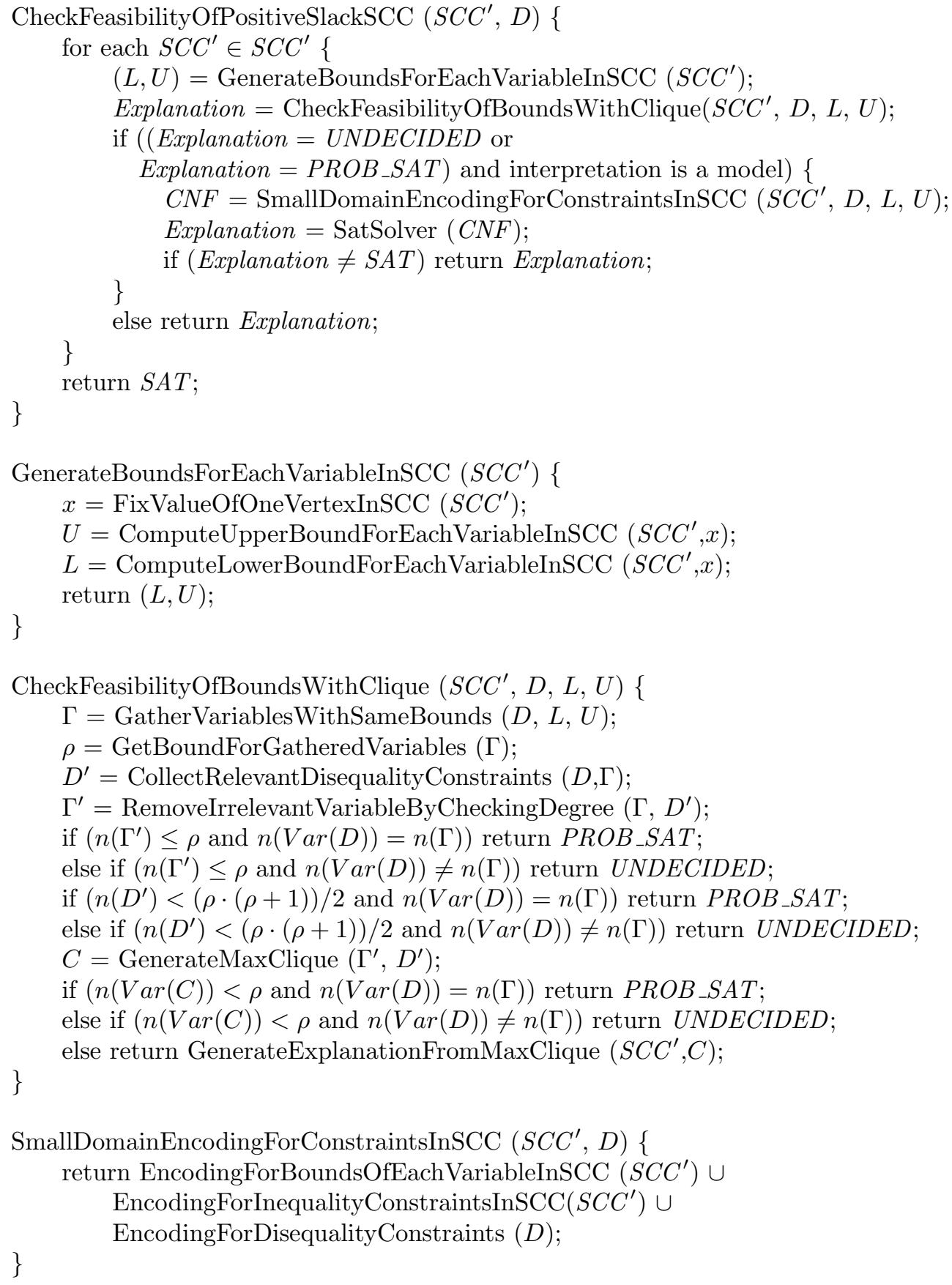

Figure 2. Theory solver algorithm (continued) 


\section{H. KIM ET AL.}

SCCs of $G_{0}$ are simply passed on to the next phase of the procedure. If no infeasibility is detected with $G_{0}$, a final feasibility check is performed by the small domain encoding method discussed in Sect. 4. For each SCC of $G$, Theorem 1 is used to compute bounds for each variable as follows.

To compute the upper bound for each variable, a variable in the SCC is chosen arbitrarily as source. (Variable $x$ in Theorem 1.) The distance from it is computed for each variable in the SCC by the Bellman-Ford algorithm. The lower bound for a variable is computed as its distance from the same source variable used to compute the upper bound after reversing the edges in the SCC. (Note that one cannot replace the distances computed by these invocations of the shortest path algorithm with those computed on $G_{a}$.)

Some inequalities and disequalities may be automatically satisfied for all values of the variables in their ranges. For instance, if $0 \leq x \leq 1$ and $2 \leq y \leq 3$, then $x \neq y$ and $y-x \leq 4$ are both satisfied. These constraints are therefore ignored in the successive steps, which consist of a quick check based on finding a clique of the disequality graph, possibly followed by propositional encoding and satisfiability check.

Some disequalities may be strengthened by converting them into a disjunction of inequalities and dropping one disjunct that is always false due to the ranges of the variables. For instance, $x-y \neq 1$, where $1 \leq x \leq 2,0 \leq y \leq 0$ can be strengthened to $x-y \geq 2$ because $x-y \leq 0$ is false for $x$ and $y$ in the given ranges. The range of $x$ therefore shrinks to $2 \leq x \leq 2$.

Example 2. Consider the SCC without any negative cycle in Fig. 3. The edges correspond to the inequality constraints $\{(x-y \leq-1),(y-x \leq 2),(z-y \leq 1),(x-z \leq-2)\}$. Additionally, there is a set of disequality constraints $\{(x-y \neq 0),(y-z \neq 0),(z-x \neq$ $0),(z-x \neq 1),(y-z \neq-1)\}$. Variable $x$ is chosen as source; hence both bounds of $x, x_{l}$ and $x_{u}$, are given value 0 . Using the Bellman-Ford algorithm, $y_{u}$ is assigned 2 and $z_{u}$ is assigned 3. Reversing the edges in the $S C C, y_{l}$ is assigned 1 and $z_{l}$ is assigned 2. Therefore, the ranges are $\{0 \leq x \leq 0,1 \leq y \leq 2,2 \leq z \leq 3\}$. The inequalities $\{(x-y \leq-1),(y-x \leq 2)\}$ and the disequalities $\{(x-y \neq 0),(z-x \neq 0),(z-x \neq 1)\}$ are automatically satisfied for all values of the variables in their ranges. The disequality $(y-z \neq 0)$ is strengthened to $(y-z \leq-1)$. Consequently, $(y-z=-1)$ and the disequality $(y-z \neq-1)$ cannot be satisfied.

The application of Theorem 2 is described in lines 53-66 of Fig. 2. We identify sets of variables for which Theorem 1 produces the same bounds and we check whether there are enough disequalities among the variables in one such set to cause inconsistency.

Specifically, suppose a set $\Gamma=\left\{\gamma_{1}, \ldots, \gamma_{p}\right\}$ of variables is found such that all variables in $\Gamma$ have the same bounds $y_{l}$ and $y_{u}$. Variables whose range is a subset of the common range are added to $\Gamma$.

Let $\rho=y_{u}-y_{l}+1$. If $|\Gamma|<\rho$, disequalities cannot cause inconsistency of this set of variables. If, on the other hand, the number of variables exceeds their common range, we check whether the disequalities form a clique of size greater than $\rho$. We first eliminate from $\Gamma$ all variables that appear in fewer than $\rho$ disequalities of the form $\gamma_{i} \neq \gamma_{j}\left(\gamma_{i}, \gamma_{j} \in \Gamma\right)$. If $\Gamma$ is not empty after this process, we greedily grow a clique, adding every time the variable appearing in the largest number of disequalities among the surviving members of $\Gamma$. This 


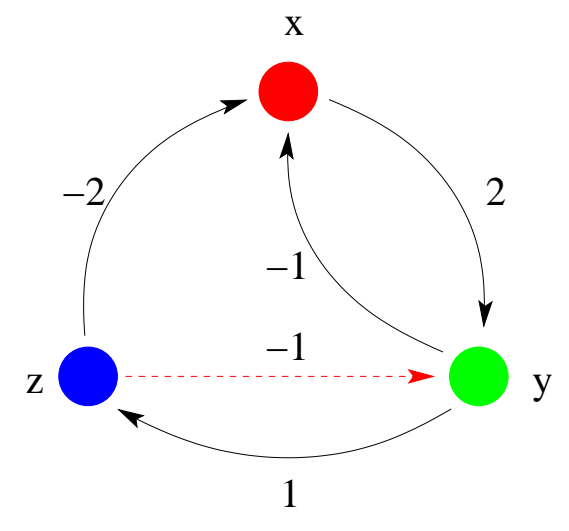

Figure 3. SCC without any negative cycle

greedy algorithm does not always find the largest clique, but is fast and works well in practice.

In the final step of the theory solver, the constraints and the bounds are converted to a set of clauses whose satisfiability is established by calling a propositional SAT solver. ${ }^{3 .}$ If the clauses are satisfiable, an interpretation for the integer variables is extracted from the solution. Otherwise, an explanation for the unsatisfiability is derived as follows from the proof of unsatisfiability returned by the SAT solver, which consists of a subset of the clauses that are found to be unsatisfiable. (The unsatisfiable core.)

Every propositional clause in the unsatisfiable core is derived from some arithmetic constraint. If a clause appears in the unsatisfiable core, the parent constraint is included in the explanation. The bound constraints on the integer variables also contribute to unsatisfiability. They are accounted for by including the constraints that form the two shortest path spanning trees found during the computation of the bounds.

Example 3. If Example 2 continues without disequality strengthening, the constraints $\{(z-$ $y \leq 1),(x-z \leq-2),(y-z \neq 0),(y-z \neq-1)\}$ and the bounds $\{0 \leq x \leq 0,1 \leq y \leq 2,2 \leq$ $z \leq 3\}$ are converted to the set of clauses below. The variable $y$ is substituted by $\iota+1$, and the variable $z$ is substituted by $\zeta+2$. As a result, the range of $\iota$ and $\zeta$ are $0 \leq \iota \leq 1$, $0 \leq \zeta \leq 1$, and the number of bits used for $\iota$ and $\zeta$ during the encoding is one instead of two.

$$
\varphi=\left(\neg \zeta_{0} \vee \iota_{0}\right) \wedge\left(\neg \iota_{0} \vee \zeta_{0}\right) \wedge\left(\neg \iota_{0} \vee \neg \zeta_{0}\right) \wedge\left(\iota_{0} \vee \zeta_{0}\right)
$$

With the set of clauses $\varphi$, a propositional SAT solver is called. Since the set of clauses is unsatisfiable, the unsatisfiable core $\Omega$ is returned:

$$
\Omega=\left(\neg \zeta_{0} \vee \iota_{0}\right) \wedge\left(\neg \iota_{0} \vee \zeta_{0}\right) \wedge\left(\neg \iota_{0} \vee \neg \zeta_{0}\right) \wedge\left(\iota_{0} \vee \zeta_{0}\right)
$$

The inequality constraints that are responsible for the bounds are extracted as an explanation from the SCC in Fig. 3. For the variables $x, y, z$ in the SCC, the edges that lie on the

3. Our current encoding of the ranges is rather unsophisticated. We are implementing a heuristic approach to minimizing the total number of encoding bits required. 
forward and backward shortest paths from each variable to the fixed variable $x$ are gathered. Therefore, we get $\{(y-x \leq 2),(z-y \leq 1),(x-z \leq-2)\}$ as an explanation for the bounds $\{0 \leq x \leq 0,1 \leq y \leq 2,2 \leq z \leq 3\}$. The parent constraints of the clauses left in $\Omega$ are finally gathered; they are $\{(z-y \leq 1),(y-z \neq 0),(y-z \neq-1)\}$. As a result, the full explanation for the infeasibility is

$$
\{(y-x \leq 2),(z-y \leq 1),(x-z \leq-2),(y-z \neq 0),(y-z \neq-1)\} .
$$

Five constraints suffice to explain the infeasibility of the original nine constraints.

\section{Related Work}

Propositional abstraction as an approach to satisfiability modulo theories was proposed in [2]. Notable solvers based on that principle are MathSAT [4, 3], ICS and Yices [8, 10], Verifun [11], BarcelogicTools [13, 23], SLICE [30], and SATORI [14]. ASAP [18] takes a dual approach, in which satisfiability of the propositional abstraction guarantees satisfiability of the original quantifier-free Presburger formula, while UCLID [20] is an eager solver. Our propositional enumeration engine is the one of $[15,16]$.

Finite instantiations for equality logic are studied in [24] and extended to difference logic in [29]; this last work has several points of contact with ours, but also important differences. The approach of [29] is eager, and the ranges are computed once and for all before invoking the propositional SAT solver. In contrast, we advocate a lazy approach and a computation of the ranges that takes place in the theory solver. Because of that, we may compute ranges more than once, but the size of the range for each variable in our algorithm is bounded by the sum of the slacks in the SCC, which is much smaller than $n+\max C$, where $\max C$ is the sum of absolute constants in the formula. In practice, ranges are much smaller in our algorithm. Moreover, we compute ranges by simply finding shortest paths in the constraint graph. The algorithm of [29], on the other hand, enumerates paths in the constraint graph and is exponential in the worst case.

Recent work by Ganai et al. [12] presents a polynomial algorithm for the computation of ranges, which improves over the one of [29], but shares the basic approach: Ranges are allocated initially, so as to be adequate for every formula built from the given set of difference constraints. Disequalities are converted to disjunctions of inequalities, instead of being retained as such in the formulation of the problem. The theory consistency problem is never converted to propositional satisfiability. Instead, range propagation allows the solver to refine the initial ranges.

MathSAT introduced the notion of layered, incremental theory solver, and that of delayed theory combination; DPLL(t) the idea of exhaustive theory propagation, both of which are included in our implementation. The importance of considering zero-slack SCCs was first pointed out in [19], which deals with RDL. Finally, [30] discusses an efficient way to implement a recursive, backtrackable Bellman-Ford algorithm.

\section{Experimental Results}

We have implemented the algorithm presented in Sect. 5 in Sateen, a theorem prover for quantifier-free first-order logic that combines the propositional reasoning engine of $[15,16]$ 


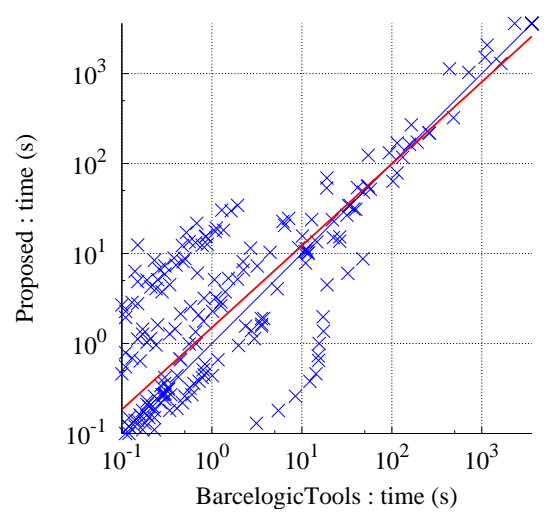

Figure 4. BarcelogicTools vs. Sateen on QFIDL

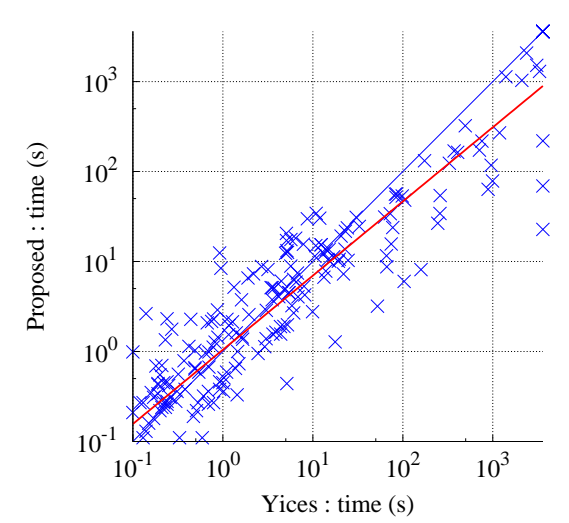

Figure 5. YICES vs. Sateen on QF IDL

with theory-specific procedures. A first set of experiments were done with the full set of QF_IDL (Quantifier free integer difference logic) benchmarks from SMT-COMP (Satisfiability Modulo Theories Competition [27]). The experiments were performed on a $1.7 \mathrm{GHz}$ Pentium 4 with 2 GB of RAM running Linux. Time out was set at 3600 seconds. Sateen was compared with BarcelogicTools [9], Yices-0.1.1 [31] and MathSAT 3.3.1 [21]. The compared solvers are the ones that were submitted to SMT-COMP in 2005.

Figures 4-6 show scatterplots comparing BarcelogicTools, Yices and MathSAT to Sateen. Points below the diagonal represent wins for Sateen. Each scatterplot shows two lines: The main diagonal, and $y=\kappa \cdot x^{\eta}$, where $\kappa$ and $\eta$ are obtained by least-square fitting. Figure 4 shows that Sateen is comparable to BarcelogicTools. In Figures 5 and 6, Sateen shows better results compared to Yices and MathSAT, especially on hard problems. The SMT-COMP benchmark formulae are such that usually the sets of constraints passed to the theory solver either contain few disequality constraints, or are such that the disequality constraints are dealt with by the zero-slack SCC algorithm. The main purpose of these experiments is therefore not to show the effectiveness of the newly proposed algorithm for finite instantiations, but to establish that Sateen is, overall, a competent solver for IDL, comparable to some of the best tools in the field.

To assess the effectiveness of the finite instantiation approach, we have generated two benchmark suites where disequality constraints play a significant role: the Queens Suite and the Job Shop Scheduling Suite. The Queens Suite contains $n$-Queens problem and $n$-SuperQueens problem. The $n$-Queens problem is a classical combinatorial search problem which consists of placing $n$ queens on a $n \times n$ board so that they do not attack each other. In the $n$-Super-Queens problem, each queen's placement is more restricted by allowing it also the knight's moves. The Job Shop Scheduling problem is a randomly generated problem which checks the feasibility of processing a number of jobs, each consisting of several tasks, on a given set of machines in a given amount of time. These two sets of benchmarks 


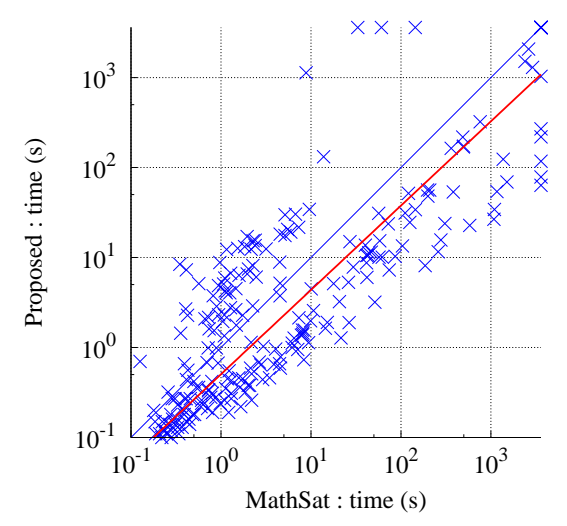

Figure 6. MATHSAT vs. Sateen on QF IDL
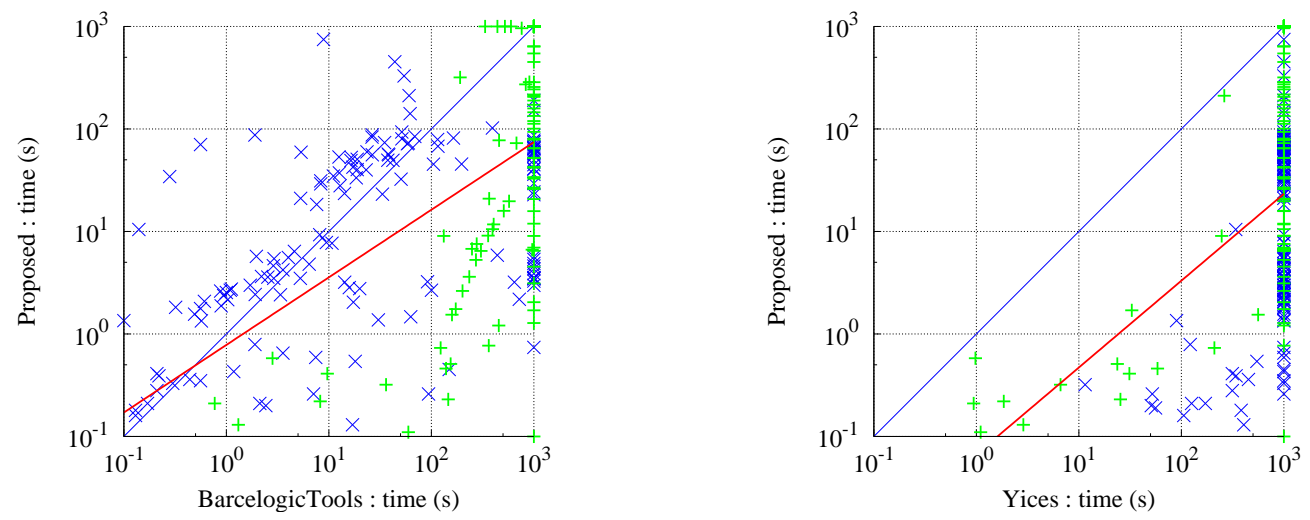

Figure 7. BarcelogicTools vs. Sateen on Figure 8. Yices vs. Sateen on Job Shop Job Shop Scheduling and Queen suites Scheduling and Queen suites

have disequality constraints that cause pigeonholing problems. In the experiment on these benchmarks, the timeout was set to 1000 seconds. ${ }^{4}$.

Figures 7-9 shows that Sateen is often orders of magnitude faster than the other solvers on these problems. The $\times$ symbols denotes the experiments on the Queens benchmarks, and the + symbols denotes the experiments on the Job Shop Scheduling benchmarks. We also provide the comparison between Sateen with our proposed algorithm and a version of Sateen that splits disequalities. Figure 10 shows that the finite instantiation algorithm works significantly better than the splitting method.

Table 1 shows the number of calls and conflicts involving the equality layer (EQ), the Bellman-Ford layer (INEQ), the zero-slack SCC layer (ZS), the clique generation layer (CLQ) and the finite instantiation layer (FI) on selected benchmarks. BV and AF correspond to the number of propositional variables and atomic formulae, respectively. In the

4. Although, the results of SMT-COMP [28] in 2006 show that Sateen is still behind the three other solvers above, Sateen gives significantly better result on the $n$-Queens and Job Shop Scheduling benchmarks. 

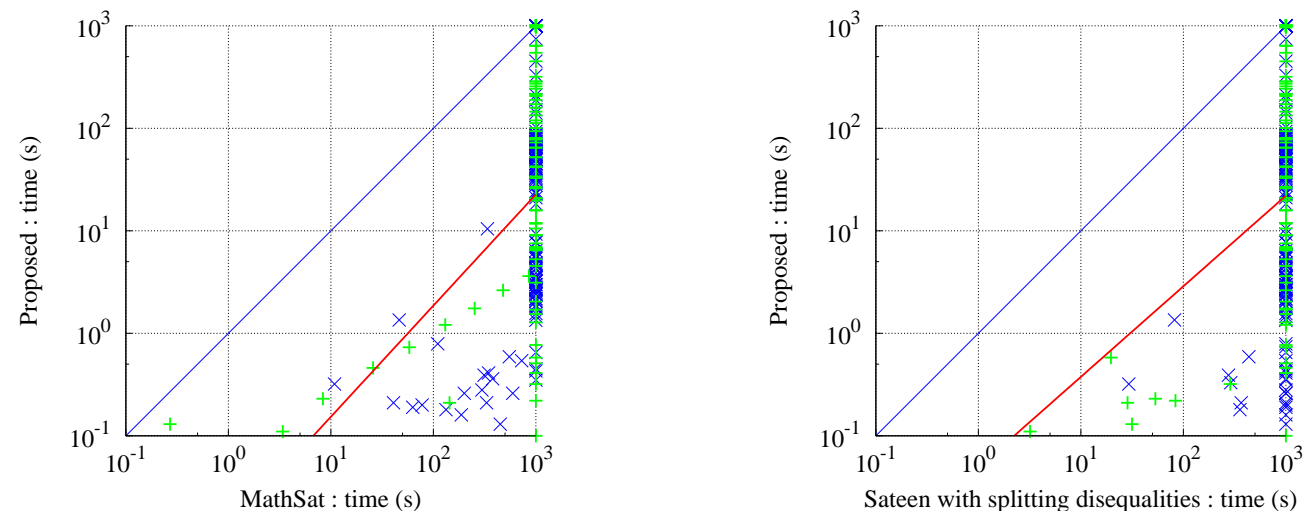

Figure 9. MathSAT vs. Sateen on Job Shop Figure 10. Sateen with splitting disequalities Scheduling and Queen suites vs. Sateen on Job Shop Scheduling and Queen suites

Table 1. Number of Calls and Conflicts

\begin{tabular}{c|cccccccc} 
Benchmark & BV & AF & SAT & EQ & INEQ & ZS & CLQ & FI \\
\hline diamonds.10.5.i.a.u & 0 & 121 & UNSAT & $0 / 0$ & $90 / 1199$ & $0 / 0$ & $0 / 0$ & $0 / 0$ \\
DTP_k2_n35_c245_s2 & 0 & 490 & SAT & $0 / 0$ & $709 / 7200$ & $0 / 0$ & $0 / 0$ & $0 / 0$ \\
inf-bakery-mutex-18 & 76 & 328 & UNSAT & $71 / 1498$ & $84 / 1533$ & $25 / 2070$ & $0 / 0$ & $0 / 0$ \\
FISCHER9-10-ninc & 1146 & 686 & SAT & $54 / 55$ & $0 / 1$ & $0 / 0$ & $0 / 0$ & $0 / 0$ \\
queen30-1 & 0 & 1365 & SAT & $0 / 0$ & $0 / 1$ & $0 / 1$ & $0 / 0$ & $0 / 1$ \\
super_queen60-1 & 0 & 5664 & SAT & $0 / 0$ & $0 / 1$ & $0 / 1$ & $0 / 0$ & $0 / 1$ \\
jobshop30-2-20-20-4-4-12 & 0 & 2820 & UNSAT & $0 / 0$ & $632 / 1264$ & $0 / 631$ & $1 / 631$ & $0 / 1$ \\
jobshop40-2-20-20-4-4-12 & 0 & 4960 & SAT & $109 / 258$ & $3 / 1343$ & $109 / 1282$ & $58 / 1172$ & $0 / 1$ \\
jobshop50-2-25-25-4-4-11 & 0 & 7700 & UNSAT & $0 / 0$ & $1802 / 3604$ & $0 / 1801$ & $1 / 1801$ & $0 / 0$ \\
jobshop60-2-30-30-4-4-12 & 0 & 11040 & SAT & $239 / 538$ & $3 / 2773$ & $239 / 2682$ & $88 / 2442$ & $0 / 1$
\end{tabular}


entries of the form $X / Y, X$ is the number of conflicts and $Y$ is the number of calls. The data show that each layer contributes to finding conflicts. In particular, the clique generation layer is very effective in finding conflicts in the Job Shop Scheduling benchmarks, which enables the solver to avoid the finite instantiation layer.

\section{Conclusions}

We have presented an approach to solving integer difference logic that is particularly effective when the constraints to be solved are rich in disequalities. By restricting consideration to a small sufficient set of solutions, we are able to compute bounds for the integer variables occurring in the constraints. Experiments indicate that this approach is more effective than splitting disequalities into the disjunction of inequalities. Further improvements in efficiency are expected from a more sophisticated encoding scheme for the finite instances that we are currently developing.

Acknowledgment The authors thank Alberto Oliveras, Alessandro Cimatti, and Leonardo de Moura for their help with BarcelogicTools, MathSAT, and Yices.

\section{References}

[1] T. Ball, R. Majumdar, T. Millstein, and S. K. Rajamani. Automatic predicate abstraction of C programs. In PLDI 01: Programming Language Design and Implementation, Snowbird, UT, June 2001.

[2] C. W. Barrett, D. L. Dill, and A. Stump. Checking satisfiability of first-order formulas by incremental translation to SAT. In E. Brinksma and K. G. Larsen, editors, Fourteenth Conference on Computer Aided Verification (CAV'02), pages 236-249. SpringerVerlag, Berlin, July 2002. LNCS 2404.

[3] M. Bozzano, R. Bruttomesso, A. Cimatti, T. Junttila, S. Ranise, P. van Rossum, and R. Sebastiani. Efficient satisfiability modulo theories via delayed theory combination. In Seventeenth Conference on Computer Aided Verification (CAV'05), pages 335-349. Springer-Verlag, Berlin, July 2005. LNCS 3576.

[4] M. Bozzano, R. Bruttomesso, A. Cimatti, T. Junttila, P. van Rossum, S. Schulz, and R. Sebastiani. An incremental and layered procedure for the satisfiability of linear arithmetic logic. In International Conference on Tools and Algorithms for Construction and Analysis of Systems (TACAS'05), pages 317-333, Edinburgh, UK, Apr. 2005. LNCS 3440.

[5] T. H. Cormen, C. E. Leiserson, and R. L. Rivest. An Introduction to Algorithms. McGraw-Hill, New York, 1990.

[6] M. Davis, G. Logemann, and D. Loveland. A machine program for theorem proving. Communications of the ACM, 5:394-397, 1962.

[7] M. Davis and H. Putnam. A computing procedure for quantification theory. Journal of the Association for Computing Machinery, 7(3):201-215, July 1960. 
[8] L. de Moura and H. Reuß. Lemmas on demand for satisfiability solvers. In Fifth International Symposium on the Theory and Application of Satisfiability Testing (SAT'02), Cincinnati, OH, May 2002.

[9] Url: http://www.lsi.upc.edu/ oliveras/bclt-main.html.

[10] B. Duterte and L. de Moura. A fast linear-arithmetic solver for DPLL(T). In Eighteenth Conference on Computer Aided Verification (CAV'06), pages 81-94, Seattle, WA, Aug. 2006. LNCS 4144.

[11] C. Flanagan, R. Joshi, X. Ou, and J. B. Saxe. Theorem proving using lazy proof explication. In W. A. Hunt, Jr. and F. Somenzi, editors, Fifteenth Conference on Computer Aided Verification (CAV'03), pages 355-367. Springer-Verlag, Berlin, July 2003. LNCS 2725.

[12] M. K. Ganay, M. Talupur, and A. Gupta. SDSAT: Tight integration of small domain encoding and lazy abstraction in a separation logic solver. In International Conference on Tools and Algorithms for Construction and Analysis of Systems (TACAS'06), pages 135-150, Vienna, Austria, Mar. 2006. LNCS 3920.

[13] H. Ganzinger, G. Hagen, R. Nieuwenhuis, A. Oliveras, and C. Tinelli. DPLL(T): Fast decision procedures. In R. Alur and D. Peled, editors, Sixteenth Conference on Computer Aided Verification (CAV'04), pages 175-188. Springer-Verlag, Berlin, July 2004. LNCS 3114.

[14] M. K. Iyer, G. Parthasarathy, and K.-T. Cheng. SATORI - a fast sequential SAT engine for circuits. In Proceedings of the International Conference on Computer-Aided Design, pages 320-325, San Jose, CA, Nov. 2003.

[15] H. Jin, H. Han, and F. Somenzi. Efficient conflict analysis for finding all satisfying assignments of a Boolean circuit. In International Conference on Tools and Algorithms for Construction and Analysis of Systems (TACAS'05), pages 287-300, Apr. 2005. LNCS 3440.

[16] H. Jin and F. Somenzi. Prime clauses for fast enumeration of satisfying assignments to Boolean circuits. In Proceedings of the Design Automation Conference, pages 750-753, Anaheim, CA, June 2005.

[17] H. Kim and F. Somenzi. Finite instantiations for integer difference logic. In Formal Methods in Computer Aided Design (FMCAD'06), pages 31-38, San Jose, CA, Nov. 2006.

[18] D. Kroening, J. Ouaknine, S. Seshia, and O. Strichman. Abstraction-based satisfiability solving of Presburger arithmetic. In R. Alur and D. Peled, editors, Sixteenth Conference on Computer Aided Verification (CAV'04), pages 308-320. Springer-Verlag, Berlin, July 2004. LNCS 3114.

[19] S. Lahiri and M. Musuvathi. An efficient Nelson-Oppen decision procedure for difference constraints over rationals. In Third International Workshop on Pragmatical 
Aspects of Decision Procedures in Automated Reasoning (PDPAR'05), pages 2-9, Edinburgh, UK, July 2005. To appear in ENTCS.

[20] S. K. Lahiri and S. A. Seshia. The UCLID decision procedure. In R. Alur and D. Peled, editors, Sixteenth Conference on Computer Aided Verification (CAV'04), pages 475478. Springer-Verlag, Berlin, July 2004. LNCS 3114.

[21] Url: http://mathsat.itc.it.

[22] M. Moskewicz, C. F. Madigan, Y. Zhao, L. Zhang, and S. Malik. Chaff: Engineering an efficient SAT solver. In Proceedings of the Design Automation Conference, pages 530-535, Las Vegas, NV, June 2001.

[23] R. Nieuwenhuis and A. Oliveras. DPLL(T) with exhaustive theory propagation and its application to difference logic. In Seventeenth Conference on Computer Aided Verification (CAV'05), pages 321-334. Springer-Verlag, Berlin, July 2005. LNCS 3576.

[24] A. Pnueli, Y. Rodeh, O. Strichman, and M. Siegel. The small model property: How small can it be? Journal of Information and Computation, 178(1):279-293, Oct. 2002.

[25] K. Ravi and F. Somenzi. Minimal assignments for bounded model checking. In International Conference on Tools and Algorithms for Construction and Analysis of Systems (TACAS'04), pages 31-45, Barcelona, Spain, Mar.-Apr. 2004. LNCS 2988.

[26] J. P. M. Silva and K. A. Sakallah. Grasp - a new search algorithm for satisfiability. In Proceedings of the International Conference on Computer-Aided Design, pages 220227, San Jose, CA, Nov. 1996.

[27] Url: http://www.csl.sri.com/users/demoura/smt-comp/.

[28] Url: http://www.csl.sri.com/users/demoura/smt-comp/results-qf_idl.shtml/.

[29] M. Talupur, N. Sinha, O. Strichman, and A. Pnueli. Range allociation for separation logic. In R. Alur and D. Peled, editors, Sixteenth Conference on Computer Aided Verification ( $C A V^{\prime} 04$ ), pages 148-161. Springer-Verlag, Berlin, July 2004. LNCS 3114.

[30] C. Wang, F. Ivancic, M. Ganai, and A. Gupta. Deciding separation logic formulae by SAT and incremental negative cycle elimination. In Logic for Programming Artificial Intelligence and Reasoning (LPAR'2005), Montego Bay, Jamaica, Dec. 2005.

[31] Url: http://fm.csl.sri.com/yices. 